\title{
Propagation of premixed isobaric flames in narrow channels with heat-losses: the asymptotic analysis revised and reliance on the flame-sheet model
}

\author{
Vadim N. Kurdyumov \\ Department of Energy, CIEMAT, Madrid, Spain
}

\begin{abstract}
A novel comprehensive analysis based on the matched asymptotic expansions method is curried out for a problem of flame propagation in narrow channels with heat-losses using the activation energy as a large parameter. The novelty consists in presentation of the results, namely the dependence of the flame velocity versus the heat-loss intensity, in a parametric form. Comparison with the numerical calculations reveals that the asymptotic results becomes in close agreement at relatively low values of the expansion parameter in such a manner extending a practical implementation of the method. The analysis may be of utility to flame-sheet model applications increasing its accuracy in general combustion studies.
\end{abstract}

\section{Introduction}

The first use of the matched asymptotic expansions method in combustion was carried out by Bush and Fendell [1] where the velocity of planar isobaric and adiabatic flames governed by the Arrhenius kinetics was approached. Despite the physical insight into the problem was advanced much before by Zel'dovich and Frank-Kamenetskii [2], the analysis presented in [1] was mathematically more rigorous involving two terms in the asymptotic expansions based on large activation energy assumption. The procedure showed a clearly visible way how the next terms in the asymptotic expansion can be obtained, at least in theory. The comprehensive discussion regarding these approaches is given in [3].

The influence of the volumetric heat losses on a freely propagating premixed flame has been understood adequately from the work by Spalding [4], where he found two different burning velocities, the slowest one of which represents an unstable solution difficult to observe in experiments. Prediction of critical heat-loss intensity above which the solution does not exist is of the utmost significance. Taking into account heat losses complicates moderately the asymptotic analysis carried out in [5, 6], see also [7].

The earliest implementation of the flame-sheet model should be traced back to Sivashinsky [8]. This approximation prompted afterwards a significant number of applications in various combustion issues. It was successfully applied to studies of diffusion-thermal instability of 
premixed flames [9], flame balls $[10,11]$ or stretched premixed flames [12]. The theoretical background for this approximation was set out in [7]. The constant-density approximation was used in all these studies: the density and transport properties changes were neglected in both the corresponding conservation equation and the reaction rate.

Consider, as an example, a planar lean flame propagating steadily in a uniform mixture at rest. In all above studies the flame-sheet approximation consisted in replacing of a finite and spatially distributed Arrhenius reaction rate,

$$
\Omega=\mathcal{B} \rho^{n} Y \exp \left\{-\frac{E}{\mathcal{R}_{g} T}\right\},
$$

by an infinitely thin reaction sheet given by

$$
\Omega=\mathcal{B}^{\prime} \exp \left\{\frac{E}{2 \mathcal{R}_{g}}\left(\frac{1}{T_{a}}-\frac{1}{T}\right)\right\} \cdot \delta\left(x^{\prime}\right) .
$$

Here $\delta(\cdot)$ is the Dirac function, $x^{\prime}$ is the (dimensional) coordinate and the flame position is situated at $x^{\prime}=0$. Notations $\rho, Y, E, \mathcal{R}_{g}, T_{a}$ and $T$ appearing in Eqs. (1)-(2) used for the density, the fuel mass fraction, the activation energy, the universal gas constant, the adiabatic temperature and the temperature, respectively. $\mathcal{B}$ and $\mathcal{B}^{\prime}$ are dimensional (appropriately defined) constant frequency factors.

For a flame propagating from right to left, integrating the mass fraction conservation equation with Eq. (2) across the flame sheet defines automatically the reactant diffusion flux to the reaction zone,

$$
-\left.\rho \mathcal{D} \frac{\partial Y}{\partial x^{\prime}}\right|_{x^{\prime}=0-}=\mathcal{B}^{\prime} \exp \left\{\frac{E}{2 \mathcal{R}_{g}}\left(\frac{1}{T_{a}}-\frac{1}{T_{f}}\right)\right\},
$$

where $\mathcal{D}$ is a diffusion coefficient and $T_{f}=T(0)$ is the flame temperature. In its turn, it leads to a direct relation between the flame velocity and $T_{f}$.

The application of the flame-sheet model has various benefits. First, the size the reaction zone is infinitely thin and, thus, limitations on the mesh size requiring a certain number of grid points inside the reaction zone become of no importance in numerical simulations. The other important advantage is a possibility of analytical solutions in some cases thus elucidating the physics of a problem, e.g. [9].

It is evident that the consumption rate should depend on the local mixture density and a general power, $n$, appears naturally in Eq. (1). There is no unanimous agreement in the combustion literature concerning the value of $n$ for which 1 or 2 were used in the majority of studies. It is remarkable that in all studies with the flame-sheet model applied the density dependence of the reaction rate was not never reflected within its $\delta$-function incarnation. 
Finally, the asymptotic investigations presented in [13] should be mentioned in connection with the above subjects. The dynamics of one-dimensional adiabatic flames, the closure problem and ways to circumvent this issue were investigated in this study, and, particularly, the effect of incomplete fuel combustion was taken into account. Unfortunately these results were not published in more accessible sources. Nevertheless it should be notified that consideration of heat-losses was not attended in [13].

The purpose of the present study is twofold. First, a novel asymptotic procedure based on the matched asymptotic expansions method is suggested and applied to a general case of the Arrhenius (lean) kinetic. Apparently, it differs from the approaches used previously where the $\beta^{-1}$-dependence for heat-losses was assumed from the start, where $\beta=E\left(T_{a}-T_{0}\right) / \mathcal{R} T_{a}^{2}$ is the Zeldovich number used as a large expansion parameter. Secondly, premixed flames propagating in narrow channels with heat-losses is investigated asymptotically and numerically. The last problem is considered within a narrow-channel approximation when the governing equations are reduced to its one-dimensional form, see, for example, [14].

The article is arranged as follows: in Section 2, the general formulation is given; the asymptotic analysis is presented in Section 3; the asymptotic results are compared with the direct numerical calculations in Section 4. Finally, conclusions are drawn in the last section.

\section{Formulation}

Consider a combustible mixture (fuel and oxidizer) at temperature $T_{0}$ flowing in a narrow channel with mean velocity $U_{0}$. In what follows, subindex " 0 " denotes initial (upstream) values. The external surface of the channel is maintained at the temperature of the fresh mixture, $T_{0}$. The heat flux through a wall is taken in regular manner being proportional to the temperature difference at each side of the wall and inversely to the wall's thickness, $d_{w}$, namely $J=-\lambda_{w}\left(T-T_{0}\right) / d_{w}$, where $\lambda_{w}$ is the (constant) wall's thermal conductivity. For the gas phase, the thermal conductivity and diffusivity are assumed to follow the law $\lambda / \lambda_{0}=\rho \mathcal{D} / \rho_{0} \mathcal{D}_{0}=\left(T / T_{0}\right)^{\sigma}$, as suggested in [15]. It is assumed also a constant effective heat capacity of the mixture, $c_{p}$, and a zero-Mach number approximation.

The mixture is considered to be deficient in fuel, so that it is enough to follow its fuel mass fraction with the upstream value $Y_{0}$. The mass fraction of the oxidizer, which is in abundance, remains nearly constant. The chemical reaction is modeled by an overall step that converts fuel to products at mass rate given by Eq. (1), namely $\Omega=\mathcal{B} \rho^{n} Y \exp \left(-E / \mathcal{R}_{g} T\right)$. Values $n=0,1,2$ will be used below thus studying different chemical models. 
The conservation equations are reduced to a one-dimensional form with flow properties averaged across the channel (narrow-channel approximation or thick flame limit). It was demonstrated that this approximation could be extended in some cases beyond its strict limit of validity [16].

The burning velocity of the corresponding planar flame, $S_{L}$, the thermal flame thickness defined as $\delta_{T}=\lambda_{0} / \rho_{0} c_{p} S_{L}$ and the adiabatic temperature of the planar flame $T_{a}=T_{0}+$ $Q Y_{0} / c_{p}$ based on the heat released per unit mass of fuel, $Q$, are used below to specify the nondimensional parameters. A non-dimensional temperature defined as $\theta=\left(T-T_{0}\right) /\left(T_{a}-T_{0}\right)$ is introduced in the following, while $Y_{0}$ and $\rho_{0}$ are used to normalize the mass fraction and the density, respectively.

Under the aforementioned assumptions, the steady dimensionless conservation equations become

$$
\begin{gathered}
\frac{d\left[\rho\left(U+u_{f}\right)\right]}{d x}=0 \\
\rho\left(U+u_{f}\right) \frac{d \theta}{d x}=\frac{d}{d x}\left[\kappa \frac{d \theta}{d x}\right]+\omega-b \theta \\
\rho\left(U+u_{f}\right) \frac{d Y}{d x}=\frac{1}{L e} \frac{d}{d x}\left[\kappa \frac{d Y}{d x}\right]-\omega, \\
\rho=(1+q \theta)^{-1}
\end{gathered}
$$

where $\kappa=(1+q \theta)^{\sigma}$ is a function of the temperature only and

$$
\omega=\frac{\beta^{2}}{2 L e u_{p}^{2}}(1+q)^{n-\sigma} \rho^{n} Y \exp \left\{\frac{\beta(\theta-1)}{1+q(\theta-1) /(1+q)}\right\}
$$

is the dimensionless reaction rate.

The traveling flame solutions are invariant with respect to the translation $x \rightarrow x+$ cont. In order to eliminate this freedom the temperature was fixed in one point, $\theta\left(x_{*}\right)=\theta_{*}$, in numerical calculations presented below without loss of generality. Notice that in the above equations written in the reference frame moving the flame velocity $u_{f}$ the quantity $U$ denotes the mean flow velocity measured with respect to the wall.

The temperature, the fuel mass fraction and the flow velocity take their prescribed values upstream,

$$
\theta=Y-1=U-m=0 \quad \text { as } \quad x \rightarrow-\infty .
$$

In the presence of heat-losses, $b>0$, the temperature must approach its initial value downstream, namely $\theta(x \rightarrow \infty)=0$. Nevertheless for small $b$, it requires excessively large domain downstream. Thus, we use the following mild conditions downstream

$$
\partial^{2} \theta / \partial x^{2}=\partial^{2} Y / \partial x^{2}=0 \quad \text { as } \quad x \rightarrow \infty
$$


The numerical simulations reported below showed that the influence of the downstream boundary conditions becomes negligible if the size of the computational domain is sufficiently large. Apart from this, Eqs. (10) are satisfied automatically for $b=0$ (the adiabatic case).

Equation (4) provides

$$
\rho\left(U+u_{f}\right)=u_{L}=\text { const }
$$

which substituted in Eqs. (5)-(6) transform them into an eigenvalue problem,

$$
\begin{aligned}
& u_{L} \frac{d \theta}{d x}=\frac{d}{d x}\left[\kappa \frac{d \theta}{d x}\right]+\omega-b \theta \\
& u_{L} \frac{d Y}{d x}=\frac{1}{L e} \frac{d}{d x}\left[\kappa \frac{d Y}{d x}\right]-\omega
\end{aligned}
$$

with the eigenvalue $u_{L}$ to be calculated. Thereafter, the upstream boundary conditions (9) allow to obtain the flame speed with respect to the wall, $u_{f}=u_{L}-m$, and, from Eq. (11), the flow velocity profile $U=u_{L} / \rho-u_{f}$. Notice, that in narrow channels the momentum conservation equation is reduced to the Darcy law, see [17], and it is not required within the zero-Mach approximation. The problem given by Eq. (12)-(13) and the corresponding boundary conditions is completely identical to that determining the velocity of a planar premixed flame with (linear) heat-losses propagating in a quiescent mixture.

The following parameters appears in the above equations: the dimensionless flow rate, $m=U_{0} / S_{L}$, the Zel'dovich number, $\beta=E\left(T_{a}-T_{0}\right) / \mathcal{R}_{g} T_{a}^{2}$, the Lewis number, Le= $\lambda_{0} / c_{p} \rho_{0} \mathcal{D}_{0}$, the heat release parameter, $q=\left(T_{a}-T_{0}\right) / T_{0}$, and the heat-loss parameter, $b=$ $\left(\delta_{T}^{2} / d_{w}\right)\left(\lambda_{w} / \lambda_{0}\right)(P / S)$, where $P$ and $S$ is the perimeter and area of the channel's cross-section, respectively. In particular, $P / S=2 / R$ for a circular channel, where $R$ is the radius.

The factor $u_{p}=S_{L} / S_{L}^{a s}$ included in Eq. (8) ensures that the non-dimensional speed of a planar adiabatic flame equals one for a given finite $\beta$, where $S_{L}^{a s}$ is the asymptotic value of adiabatic laminar flame speed calculated at $\beta \rightarrow \infty$,

$$
S_{L}^{a s}=\sqrt{2\left(\lambda_{0} / c_{p}\right) L e \beta^{-2} \mathcal{B} \rho_{0}^{n-2}\left(T_{a} / T_{0}\right)^{\sigma-n}} \exp \left(-E / 2 \mathcal{R} T_{a}\right)
$$

Adequate calculation of $u_{p}$ requires the solution of the following eigenvalue problem

$$
\begin{aligned}
& d \theta / d z=d[\kappa(\theta) d \theta / d z] / d z+\omega, \quad d Y / d z=L e^{-1} d[\kappa(\theta) d Y / d z] / d z-\omega \\
& z \rightarrow-\infty: \quad \theta=Y-1=0, \quad z \rightarrow+\infty: \quad \theta-1=Y=0 .
\end{aligned}
$$

The numerical values of $u_{p}$ calculated by a shotting method are plotted in Fig. 1 as functions of the Zeldovich number $\beta$ (left plot) and the Lewis number Le (right plot) for various values of $n$ and for $\sigma=0$ and 0.7. It is important that, according to [1], the eigenvalue of Eq. (15) is of the form $u_{p}=1+a_{1} / \beta+\ldots$, as $\beta \rightarrow \infty$. Thus, it does not affect the asymptotic results exposed below within the approximation considered. 

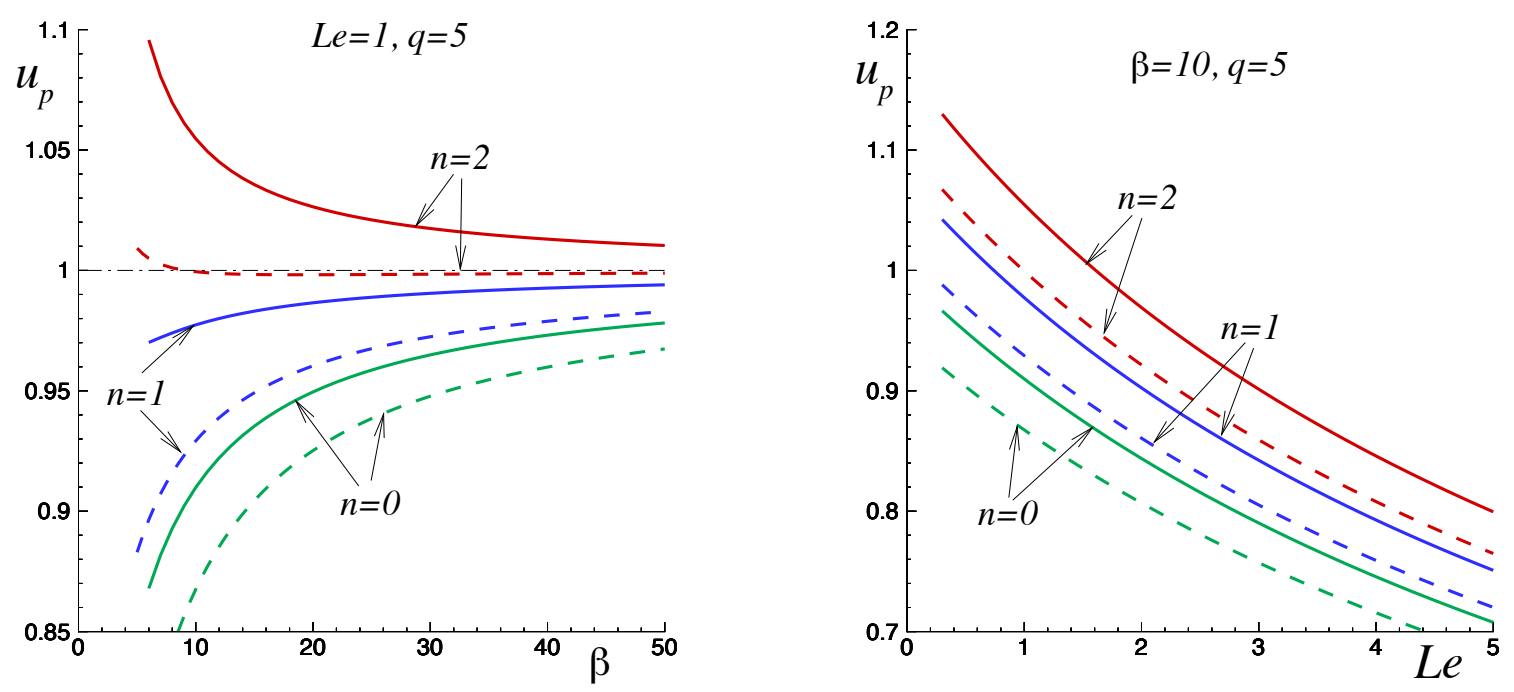

Figure 1: Numerical values of the factor $u_{p}=S_{L} / S_{L}^{a s}$ appearing in Eq. (8) plotted as a function of the Zeldovich number, $\beta$ (left plot), and the Lewis number, $L e$ (right plot), for various values of the density power $n$; solid lines $-\sigma=0$, dashed lines $-\sigma=0.7$.

\section{Asymptotic analysis}

The under-exponential expression in the reaction-rate term (8) is recast as follows

$$
\frac{\beta(\theta-1)}{1+q(\theta-1) /(1+q)}=A+\frac{\tilde{\beta}\left(\theta-\theta_{f}\right)}{1+\tilde{q}\left(\theta-\theta_{f}\right) /(1+\tilde{q})},
$$

where $\theta_{f}$ is the flame temperature, unknown beforehand, and

$$
A=\frac{\beta\left(\theta_{f}-1\right)}{1+q\left(\theta_{f}-1\right) /(1+q)}, \quad \tilde{\beta}=\frac{\beta}{\left[1+q\left(\theta_{f}-1\right) /(1+q)\right]^{2}}, \quad \tilde{q}=\frac{q}{1+q\left(\theta_{f}-1\right) /(1+q)} .
$$

The similar transformation was used in [17] to study the premixed flames in long, narrow, adiabatic channels in the presence of gas compressibility.

Using Eq. (16), the reaction-rate term (8) becomes

$$
\omega=(1+q)^{n-\sigma} \rho^{n}\left(\theta_{f}\right) \frac{\beta^{2}}{\tilde{\beta}^{2}} e^{A} \cdot \frac{1}{2 \operatorname{Leu} u_{p}^{2}} \frac{\rho^{n}(\theta)}{\rho^{n}\left(\theta_{f}\right)} Y \exp \left\{\frac{\tilde{\beta}\left(\theta-\theta_{f}\right)}{1+\tilde{q}\left(\theta-\theta_{f}\right) /(1+\tilde{q})}\right\}
$$

Let us re-scale the length as $\xi=u_{L} x / \kappa\left(\theta_{f}\right)$. Introducing the following parameters

$$
\mu=\frac{(1+q)^{n-\sigma} \rho^{n}\left(\theta_{f}\right) \kappa\left(\theta_{f}\right)}{u_{L}^{2}} \frac{\beta^{2}}{\tilde{\beta}^{2}} e^{A}=\frac{1}{u_{L}^{2}} \frac{(1+q)^{4+n-\sigma}}{\left(1+q \theta_{f}\right)^{4+n-\sigma}} e^{A}, \quad \tilde{b}=\frac{b \kappa\left(\theta_{f}\right)}{u_{L}^{2}},
$$


allows to rewrite Eq. (12)-(13) in the form

$$
\begin{aligned}
& \frac{d \theta}{d \xi}=\frac{d}{d \xi}\left[\frac{\kappa(\theta)}{\kappa\left(\theta_{f}\right)} \frac{d \theta}{d \xi}\right]+\tilde{\omega}-\tilde{b} \theta, \\
& \frac{d Y}{d \xi}=\frac{1}{L e} \frac{d}{d \xi}\left[\frac{\kappa(\theta)}{\kappa\left(\theta_{f}\right)} \frac{d Y}{d \xi}\right]-\tilde{\omega} .
\end{aligned}
$$

where

$$
\tilde{\omega}=\mu \frac{\tilde{\beta}^{2}}{2 L e u_{p}^{2}} \frac{\left(1+q \theta_{f}\right)^{n-\sigma}}{(1+q \theta)^{n}} Y \exp \left\{\frac{\tilde{\beta}\left(\theta-\theta_{f}\right)}{1+\tilde{q}\left(\theta-\theta_{f}\right) /(1+\tilde{q})}\right\} .
$$

The solution of Eq. (19)-(20) with the boundary conditions Eqs. (9)-(10) determines the flame temperature, $\theta_{f}$, and the value of $\mu$, both functions of parameter $\tilde{b}$. Then, using Eq. (18), the flame velocity and the heat-loss parameter are expressed as follows

$$
u_{L}=\mu^{-1 / 2}\left(\frac{1+q \theta_{f}}{1+q}\right)^{2+(\sigma-n) / 2} \exp \left\{\frac{\beta}{2} \cdot \frac{\left(\theta_{f}-1\right)}{\left(1+q \theta_{f}\right) /(1+q)}\right\}, \quad b=\tilde{b} \frac{u_{L}^{2}}{\left(1+q \theta_{f}\right)^{\sigma}},
$$

where $\tilde{b}$ plays role of a parameter. One can see that the variation of $b$ with $\beta$ turns out more complex than the dependence $b \sim 1 / \beta$ imposed usually from the start in previous asymptotic studies.

The flame is situated at $\xi=0$ without loss of generality. As usual in the limit $\tilde{\beta} \gg 1$, the thickness of the reaction rate zone is of order $O\left(\tilde{\beta}^{-1}\right)$ outside of which $\tilde{\omega}$ remains exponentially small. It suggests $\tilde{\omega}=0$ in two outer regions extending from $\xi=0$ to $\xi= \pm \infty$. The thin inner region is however characterized by a nonzero $\tilde{\omega}$ where both the temperature and the mass fraction undergo small variations. In the following, the matched asymptotic expansions method is applied using $\tilde{\beta}$ as a large parameter and leading order results are derived.

\subsection{Outer solution}

In the ordinary way, Eqs. (19)-(20) produce a standard jump condition across the reaction zone,

$$
\left\|\frac{d \theta}{d \xi}\right\|+\frac{1}{L e}\left\|\frac{d Y}{d \xi}\right\|=0
$$

obtained by summing them and integrating across the reaction region, where $\|f\|$ denotes $f(0+)-$ $f(0-)$. In the strict sense, it should be confirmed by the inner-region analysis given below.

Imposing $\tilde{\omega}=0$ in the outer region, Eq. (20) has the first integral

$$
Y-\frac{1}{L e} \frac{\kappa(\theta)}{\kappa\left(\theta_{f}\right)} \frac{d Y}{d \xi}=\text { const }
$$


Anticipating the matching conditions, $Y=O\left(\tilde{\beta}^{-1}\right)$ is required in the inner region. Then, using Eqs. (9) and (23) one gets, to leading order,

$$
\lim _{\xi \rightarrow 0-} \frac{1}{L e} \frac{d Y}{d \xi}=-1, \quad \text { and } \quad \lim _{\xi \rightarrow 0+} \frac{1}{L e} \frac{d Y}{d \xi}=0
$$

where $\left.\theta\right|_{\xi=0}=\theta_{f}$ was applied. Finally, the temperature in the outer regions is governed by the only equation

$$
\frac{d \theta}{d \xi}=\frac{d}{d \xi}\left[\left(\frac{1+q \theta}{1+q \theta_{f}}\right)^{\sigma} \frac{d \theta}{d \xi}\right]-\tilde{b} \theta,
$$

to be solved subject the the following jump and boundary conditions

$$
\|\theta\|=0, \quad\left\|\frac{d \theta}{d \xi}\right\|+1=0, \quad \theta(\xi \rightarrow \pm \infty)=0 .
$$

Let us denote $\Phi^{( \pm)}=d \theta /\left.d \xi\right|_{\xi \rightarrow \pm 0}$. The solution of Eqs. (25)-(26) provides the flame temperature $\theta_{f}$ and $\Phi^{( \pm)}$as functions of $\tilde{b}$.

The problem given by Eqs. (25)-(26) is nonlinear and does not possess exact analytical solution for $\sigma>0$. The matter is simplified much for $\sigma=0$. In this case Eqs. (25)-(26) have an analytic solution in the form

$$
\theta= \begin{cases}\theta_{f} \exp \left\{a^{(-)} \xi\right\}, & \xi<0 \\ \theta_{f} \exp \left\{a^{(+)} \xi\right\}, & \xi>0\end{cases}
$$

where

$$
a^{(-)}=\frac{1+\sqrt{1+4 \tilde{b}}}{2}, \quad a^{(+)}=\frac{1-\sqrt{1+4 \tilde{b}}}{2} .
$$

Thus,

$$
\theta_{f}=(1+4 \tilde{b})^{-1 / 2}, \quad \Phi^{( \pm)}=\left[\theta_{f} \mp 1\right] / 2 .
$$

An approximate solution is developed in Appendix A for arbitrary $\sigma$ as series in power of $\epsilon=q /(1+q)$.

\subsection{Inner solution}

As usual, the solution in the reaction zone requires stretching of the coordinate, by writing $\zeta=\tilde{\beta}^{-1} \xi$, and perturbing all variables relative to their values at $\tilde{\xi}=0$, namely

$$
\theta=\theta_{f}-\tilde{\beta}^{-1} \phi(\zeta)+\ldots, \quad Y=\tilde{\beta}^{-1} \psi(\zeta)+\ldots
$$

The corresponding matching conditions between the inner and outer solutions are

$$
\lim _{x \rightarrow \pm 0} \frac{d \theta}{d x}=-\lim _{\zeta \rightarrow \pm \infty} \frac{d \phi}{d \zeta}, \quad \lim _{x \rightarrow \pm 0} \frac{d Y}{d x}=\lim _{\zeta \rightarrow \pm \infty} \frac{d \psi}{d \zeta}
$$




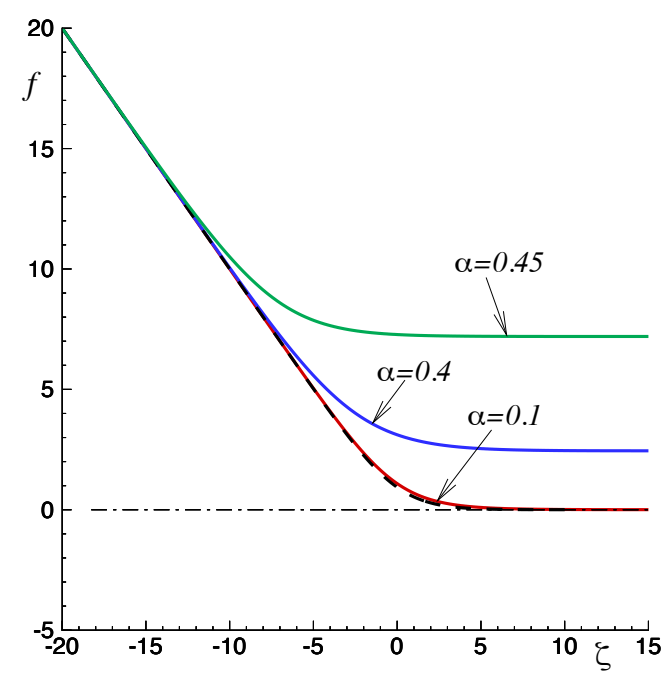

Figure 2: Distributions of $f(\zeta)$ calculated for various $\alpha$; the solution for $\alpha=0$ is plotted with a dashed curve.

On substitution Eq. (29) into the governing equations, one finds, to leading order $O(\tilde{\beta})$,

$$
\frac{d^{2} \phi}{d \zeta^{2}}=\frac{1}{2 L e} \mu \psi e^{-\phi}, \quad \frac{1}{L e} \frac{d^{2} \psi}{d \zeta^{2}}=\frac{1}{2 L e} \mu \psi e^{-\phi} .
$$

The following first integral is handled

$$
\phi-\frac{1}{L e} \psi=\alpha \zeta
$$

where a constant term is not included in the right-hand side of Eq. (32) because it can be incorporated in $\mu$.

The general rules of the matched asymptotic expansions method give the matching conditions:

$$
\frac{d \phi}{d \zeta} \rightarrow\left\{\begin{array} { l l } 
{ - \Phi ^ { ( - ) } , , } & { \zeta \rightarrow - \infty , } \\
{ - \Phi ^ { ( + ) } , } & { \zeta \rightarrow + \infty , }
\end{array} \quad \frac { 1 } { L e } \frac { d \psi } { d \zeta } \rightarrow \left\{\begin{array}{ll}
-1, & \zeta \rightarrow-\infty \\
0, & \zeta \rightarrow+\infty
\end{array}\right.\right.
$$

One can see that the jump condition (22) is satisfied automatically.

Finally, Eq. (32)-(33) provide

$$
\begin{cases}-\Phi^{(-)}+1 & =\alpha \\ -\Phi^{(+)} & =\alpha\end{cases}
$$

Particularly for $\sigma=0$, it leads to

$$
\frac{1-\theta_{f}}{2}=\alpha .
$$



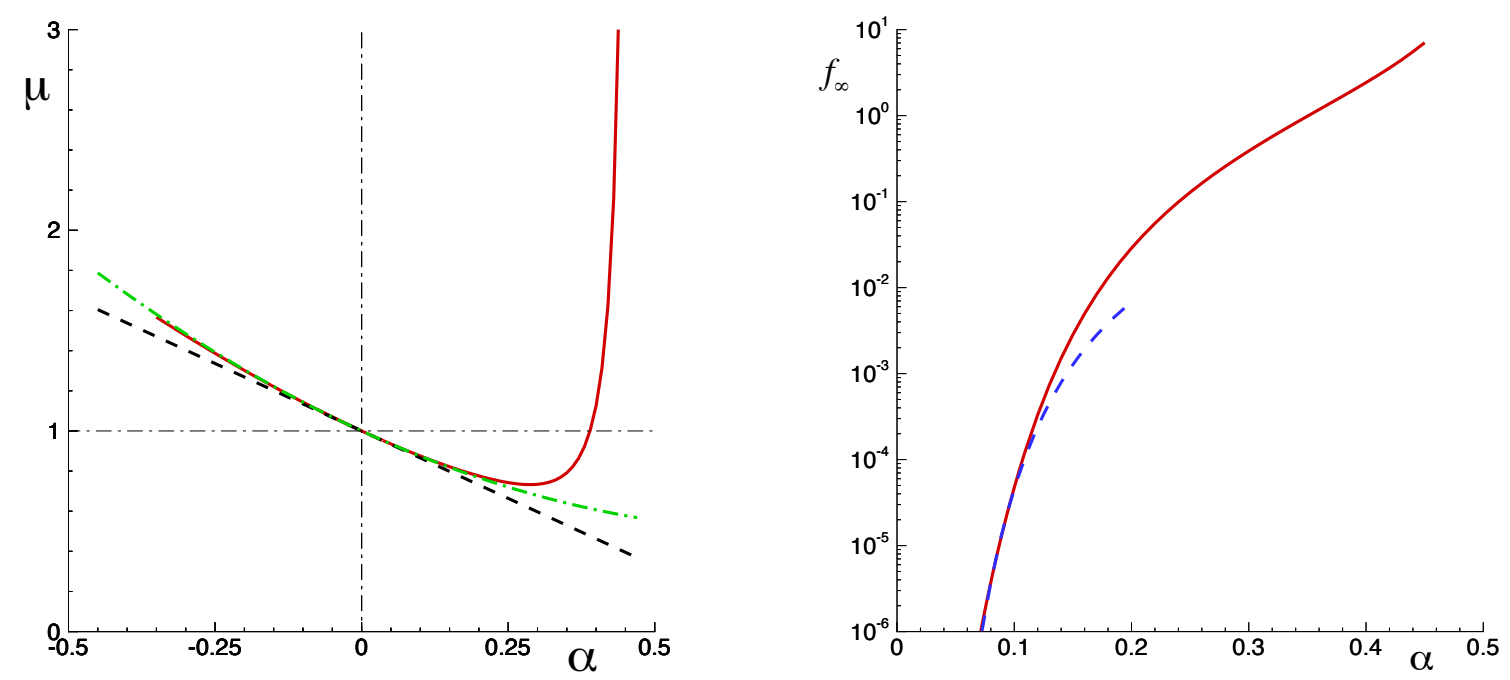

Figure 3: Variations of $\mu$ (left) and $f_{\infty}$ (right) with $\alpha$ are plotted with solid lines; dashed and dash-dot lines (left plot) show the asymptotic behaviors $\mu=1-\mu_{1} \alpha$ and $\mu=1-\mu_{1} \alpha-$ $\mu_{2} \alpha^{2}$, respectively, plotted with $\mu_{1} \approx 1.344046$ and $\mu_{2} \approx-0.90323$ calculated for small $\alpha$ in Appendix B; a dashed curve in the right plot shows the asymptotic behavior $f_{\infty}=e^{-1 / \alpha}$ at $\alpha \rightarrow 0+$.

Let us denote $f=L e^{-1} \psi$. Equations (31) are reduced to the only equation

$$
\frac{d^{2} f}{d \zeta^{2}}=\frac{1}{2} \mu f e^{(-f-\alpha \zeta)},
$$

to be solved subject to the boundary conditions

$$
\zeta \rightarrow-\infty: \quad f \rightarrow-\zeta ; \quad \zeta \rightarrow+\infty: \quad \frac{d f}{d \zeta}=0
$$

The problem given by Eqs. (36)-(37) represents a classical problem formulated and solved first in the Liñán's pioneering study of non-premixed flames [18]. The only distinction between the current and Liñán's formulations is that the shift was used in [18] for the asymptotic behavior $f(\zeta \rightarrow-\infty)=-\zeta+\delta$, where $\delta$ was an eigenvalue of the problem instead of $\mu$. The rest of the present section is given below for the sake of completeness.

The solution of Eqs. (36)-(37) determines $\mu$ and $f_{\infty}=f(\zeta \rightarrow \infty)$ as functions of $\alpha$. The last value determines an amount of fuel leakage, $Y(\xi \rightarrow \infty)=\tilde{\beta}^{-1} L e f_{\infty}$, which is of the next order in $\tilde{\beta}$. Evidently, the range $\alpha<1 / 2$ should be considered in order to have positive $\theta_{f}$. Negative values of $\alpha$ correspond to super-adiabatic flames, $\theta_{f}>1$, not considered in the present study. 
It is easily seen from Eq. (36)-(37) that $f_{\infty}=0$ is required for $\alpha \leq 0$. Indeed, otherwise the zeroth derivative condition is not asserted as $\zeta \rightarrow+\infty$ owing to non-vanishing of the right-hand side of Eq. (36).

Instead of using a numerical fitting suggested in [18], Eqs (36)-(37) were solved numerically using two methods. The first one was a standard shooting method integrating Eq. (36) from right to left. The second method was applied only for $\alpha>0$. Let us consider the transformation

$$
t=\frac{\sqrt{2 \mu}}{\alpha} e^{-\alpha \zeta / 2}, \quad g=f / f_{\infty}
$$

where the limits $\zeta=\infty$ and $\zeta=-\infty$ correspond to $t=0$ and $t=\infty$, respectively, for $\alpha>0$. The substitution of Eq. (38) into equations (36)-(37) gives

$$
\begin{gathered}
d^{2} g / d t^{2}+t^{-1} d g / d t=g e^{-f_{\infty} g}, \\
g(0)=1, \quad g(t \rightarrow \infty)=\infty .
\end{gathered}
$$

Equations (39) have the only parameter $f_{\infty}$. It is easily seen that $g \rightarrow C_{1} \ln t-C_{2}$ as $t \rightarrow \infty$ with $C_{1}$ and $C_{2}$ being functions of $f_{\infty}$. Rewriting it in terms of $\zeta$ produces the following relations corresponding to the limit $f(\zeta \rightarrow-\infty)=-\zeta$ :

$$
\alpha=\frac{2}{f_{\infty} C_{1}}, \quad \mu=\frac{1}{2} \alpha^{2} \exp \left(\frac{2 C_{2}}{C_{1}}\right)
$$

These relations were used to calculate $\alpha$ and $\mu$. A shooting procedure is not required to calculate $\alpha$ and $\mu$ for a given value of $f_{\infty}$. The values of $C_{1}$ and $C_{2}$ are presented in Appendix B.

Figure 2 shows typical distributions of $f(\zeta)$ calculated for various $\alpha$. Figure 3 displays the quantities $\mu$ and $f_{\infty}$ plotted as functions of $\alpha$. One can see that $f_{\infty}$ are small but always nonzero for positive $\alpha$. It can be demonstrated also that $f_{\infty}=e^{-1 / \alpha}$ as $\alpha \rightarrow 0+$ shown in Fig. 3 (right) with a dashed line.

It is important for practical implementations that for moderately small values of $|\alpha|$ the dependence $\mu$ on $\alpha$ remains practically linear, $\mu \approx 1-\mu_{1} \alpha$, where $\mu_{1}=1.344046$ was calculated numerically. The similar value was obtained in [18]. This linear dependence is shown in Fig. 3 (left) with a dashed line.

\section{Comparison of asymptotic and numerical results}

\subsection{Numerical solutions}

Solutions of Eqs. (12)-(13) were calculated numerically for comparison with the asymptotic results. It is common knowledge that the response curve $u_{L}$ versus $b$ has two branches, the upper 

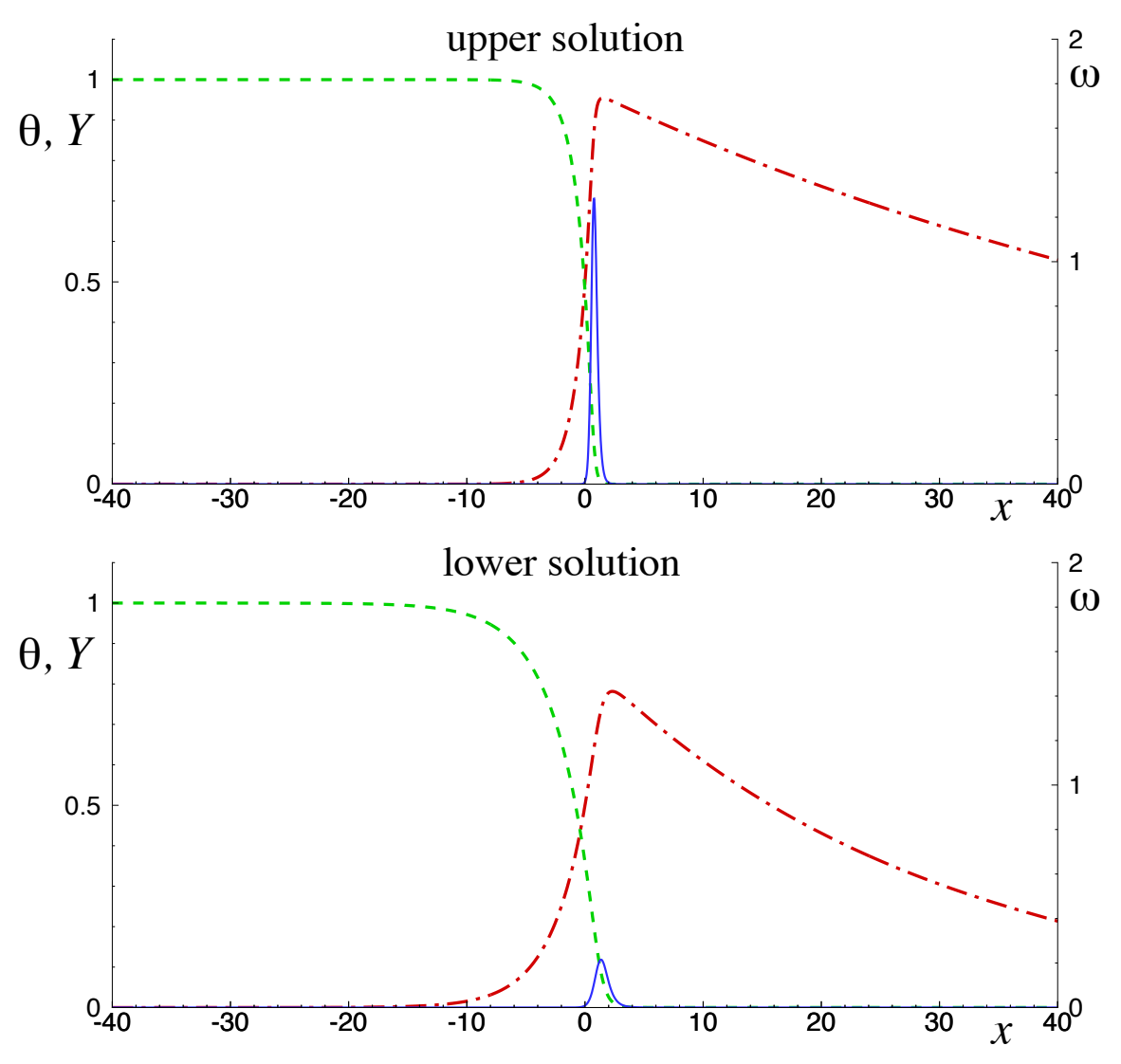

Figure 4: Spatial profiles of the reaction rate, $\omega$ (solid lines), the temperature, $\theta$ (dash-dotted lines), and the fuel mass fraction, $Y$ (dashed lines) calculated for $\beta=10, L e=1, q=5, n=2$ and $b=0.012$ corresponding to the upper and lower branches of the $\mathrm{C}$-shaped response curve; for the upper solution $u_{f} \approx 0.831$ and for the lower solution $u_{f} \approx 0.312$.

and the lower ones. Two typical profiles of the temperature, mass fraction and the reaction rate are plotted in Fig. 4 for $\beta=10, L e=1, q=5, n=2$, both calculated with $b=0.012$. It is well known that the solutions from the lower parts of the response curve are unconditionally unstable and, thus, the upper parts hold much practical significance.

The dimensionless velocity $u_{L}$ is shown in Fig. 5 (left) as a function of the heat-loss parameter $b$ for $n=2, q=5$ and various $\beta$ and $L e$ representing typical C-shaped curves. The solid curves correspond to $L e=1$, the dash-dotted curves with diamond symbols - to $L e=1.5$ and the dashed curves with triangles - to $L e=0.5$. All curves go through the point $b=0, u_{L}=1$ by virtue of $u_{p}$ introduced in Eq. (8).

Figure 5 shows that the curves calculated with different $L e$ are merging into one with increasing $\beta$. It means that the leading term in the corresponding asymptotic expansion becomes 

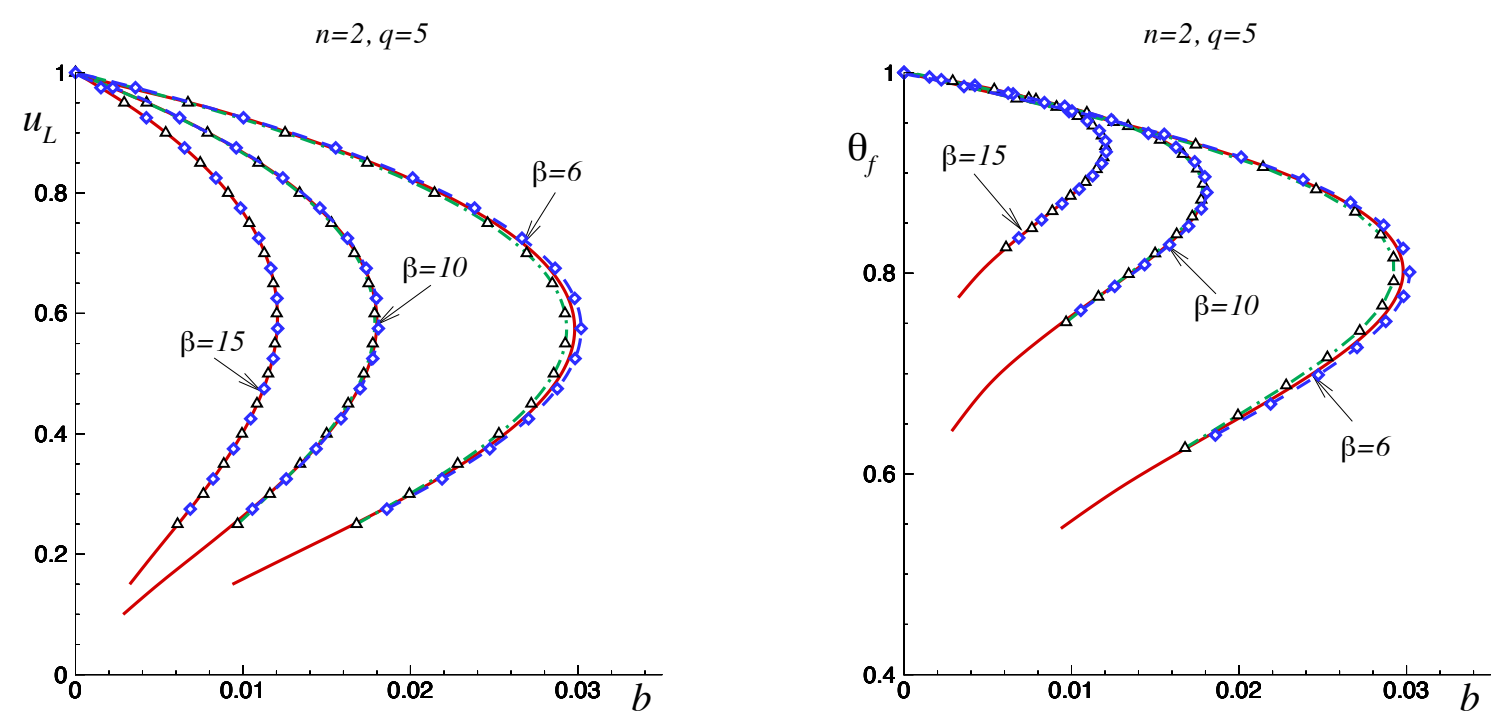

Figure 5: Computed flame velocity $u_{L}$ (left plot) and the flame temperature (right plot, the maximum temperature in Fig. 4) as functions of the heat-loss parameter $b$ for $q=5, n=2$ and various $\beta$ and $L e$ : solid lines $-L e=1$; dash-dotted lines with diamonds symbols $-L e=1.5$; dashed lines with triangles $-L e=0.5$.

independent on the Lewis number. This conclusion is supported by the asymptotic analysis presented in the previous section. Indeed, the flame temperature, $\theta_{f}$, determined by Eq. (25)-(26) is independent on $L e$. Once $\theta_{f}$ is calculated, the parametric dependence $u_{L}$ versus $b$ given by Eq. (21) remains also independent on the Lewis number.

The temperature profiles illustrated in Fig. 4 have a maximum value for $b>0$ which is associated with the flame temperature, $\theta_{f}$. It is plotted in Fig. 5 (right) as function of $b$ where the curves also merge with increasing $\beta$, as it should be. The useful observation from Fig. 5 is that all turning points have the flame temperature $\theta_{f}$ above 0.8 . According to Eq. (35) it corresponds to $\alpha \lesssim 0.1$. Figure 3 shows that within this segment $\mu(\alpha) \approx 1-\mu_{1} \alpha$ with acceptable accuracy. Namely this segment corresponds to the upper part of the C-shaped curves including the turning point. 


\subsection{Comparison of results}

In general cases $\sigma \neq 0$, the procedure required to calculate the flame velocity versus heat-losses is

$$
\tilde{b} \Rightarrow \underbrace{\left\{\theta_{f}(\tilde{b}), \alpha=-\Phi^{(+)}(\tilde{b})\right\}}_{\text {Outer problem, Eqs. (25)-(26) }} \Rightarrow \underbrace{\mu(\alpha) \approx 1-\mu_{1} \alpha}_{\text {Inner problem, Eqs. (36)-(37) }} \Rightarrow \underbrace{\left\{u_{L}, b=u_{L}^{2} \tilde{b} /\left(1+q \theta_{f}\right)^{\sigma}\right\}}_{\text {Eq. (21) }}
$$

For $\sigma=0$, the steps are reduced, by virtue of Eqs. (28) and (35), to the equation

$$
b=\frac{1}{4}\left(\frac{1}{\theta_{f}^{2}}-1\right) u_{L}^{2}\left(\theta_{f}\right),
$$

where $u_{L}\left(\theta_{f}\right)$ is given by Eq. (21). Equation (42) determines implicitly the flame temperature $\theta_{f}$ as a function of $b$ thus determining $u_{L}$ throughout Eq. (21).

The right-hand side of Eq. (42) depends on $\beta$ reproducing in the limit $\beta \rightarrow \infty$ the classical result, see $[5,6]$. Indeed, let us assume $\theta_{f}=1-c / \beta$ as $\beta \rightarrow \infty$. To leading order, Eqs. (21) and (42) give

$$
u_{L}=e^{-c / 2}+O\left(\beta^{-1}\right), \quad b=\frac{1}{\beta} \cdot \frac{1}{2} c e^{-c}+O\left(\beta^{-1}\right) .
$$

where parameters $q, n$ vanishes in first approximation. Eliminating $c$ produces the well known relation

$$
b=\frac{1}{\beta} u_{L}^{2} \ln \left(1 / u_{L}\right) .
$$

The distinction between the asymptotic results given by Eq. (42) and (44) lies in its applicability. While Eq. (44) is applicable as $\beta \rightarrow \infty$, the asymptotic dependence given by Eqs. (21) and (42) is valid also for moderately large $\beta$ thus extending the practicality of the asymptotic results. It is illustrated below comparing the asymptotic and exact (numerical) curves.

Figure 6 shows the numerical response curves based on Eqs. (12)-(13) (solid lines), the asymptotic results given by Eqs. (42) and Eq. (21) (dashed lines) and the limiting curve given by Eq. (44) (dash-dot-dot lines) plotted for $\sigma=0, \beta=10, q=5, L e=1, n=1$ (left) and $n=2$ (right). The asymptotic curves plotted with $\mu=1$ in Eq. (21) are shown with dash-dot lines. One can see that the asymptotic curves (dashed lines) approach much better the exact curves (solid lines) at a relatively low value of $\beta$. This figure reveals also an importance of the correction coming from the inner region, namely $\mu(\alpha) \approx 1-\mu_{1} \alpha$. One can see that the curves corresponding to $\mu \equiv 1$ (dash-dot lines) are visibly farther from the exact (numerical) curves. An agreement between the numerical and asymptotic results increases, as it should be, with increasing $\beta$. Figure 7 shows the curves calculated with $\beta=15, q=5, L e=1$ and $n=0,1,2$.

The same trend was observed for $\sigma>0$ when the direct relation between $\theta_{f}$ and $\alpha$ similar to Eq. (35) does not exist. In these cases the five-terms expansion presented in Appendix A was 

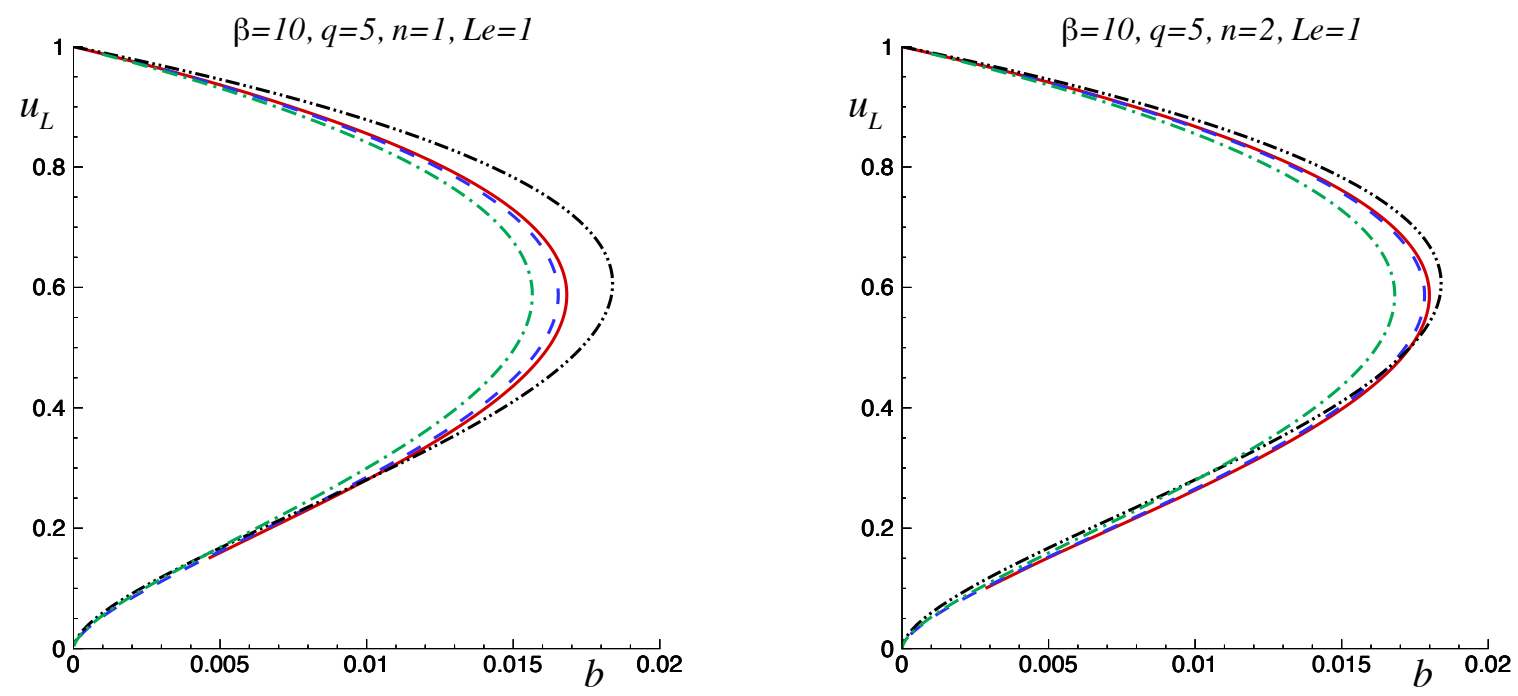

Figure 6: Flame velocity $u_{L}$ versus $b$ plotted for $\sigma=0, \beta=10, q=5, L e=1, n=1$ (left plot) and $n=2$ (right plot): solid lines - direct numerical calculations; dashed lines the asymptotic given by Eqs. (21) and (42); dash-dot lines - the asymptotic results plotted with $\mu=1$; dash-dot-dot lines - the expression $b=\beta^{-1} u_{L}^{2} \ln \left(1 / u_{L}\right)$.

used to calculated $\theta_{f}$ and $\Phi^{(+)}$. The steps expressed by Eq. (41) were carried out. Figure 8 shows $u_{L}$ plotted versus $b$ for $\sigma=0.7, q=5, n=1,2$ and two values of $\beta$.

It is of interest to acquire the limit $\beta \rightarrow \infty$ for distinct from zero $\sigma$ similar to that given by Eq. (44) for $\sigma=0$. Let us assume again that

$$
\theta_{f}=1-\frac{c}{\beta}, \quad \text { as } \quad \beta \rightarrow \infty
$$

which leads again to $u_{L}=e^{-c / 2}+o(1)$.

On the other hand, expansion (A4) provides

$$
\tilde{b} \rightarrow 0: \quad \theta_{f}=1-A(\sigma, q) \cdot \tilde{b}+O\left(\tilde{b}^{2}\right)+\ldots, \quad \Phi^{(+)}=-\tilde{b}+O\left(\tilde{b}^{2}\right)+\ldots
$$

where

$$
A(\sigma, q)=2-\frac{(1+q)^{-\sigma}+q \sigma-1}{q(\sigma+1)}
$$

is a coefficient calculated in Appendix A written in terms of $q$. Combination of Eqs. (41), (45) and (46) gives, to leading order,

$$
b=\frac{1}{\beta} \cdot \frac{2}{A(\sigma, q)(1+q)^{\sigma}} u_{L}^{2} \ln \left(1 / u_{L}\right) .
$$




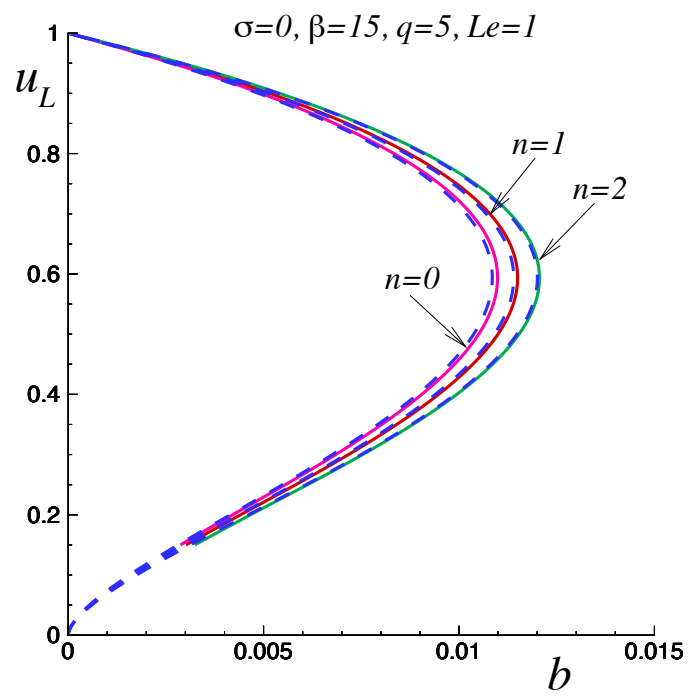

Figure 7: Flame velocity $u_{L}$ versus $b$ plotted for $\sigma=0, \beta=15, q=5, L e=1, n=1$ and $n=0,1,2$ : solid lines - direct numerical calculations; dashed lines - the asymptotic given by Eqs. (21) and (42).
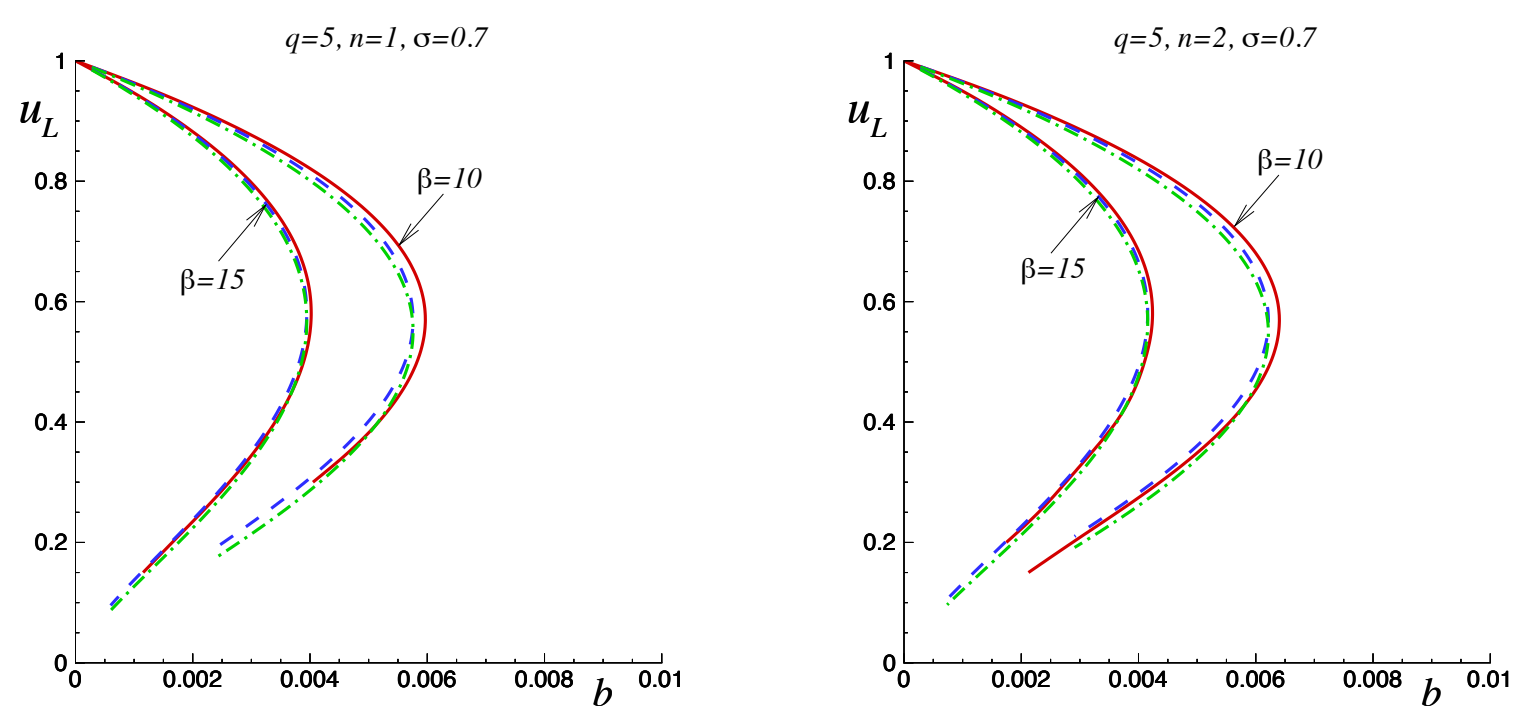

Figure 8: Flame velocity $u_{L}$ versus $b$ plotted for $\sigma=0.7, q=5$ and various $\beta$; the left plot - $n=1$, the right plot - $n=2$; solid lines - the numerical results; dashed lines - asymptotic results; dash-dotted lines - the asymptotic results plotted with $\mu \equiv 1$. 


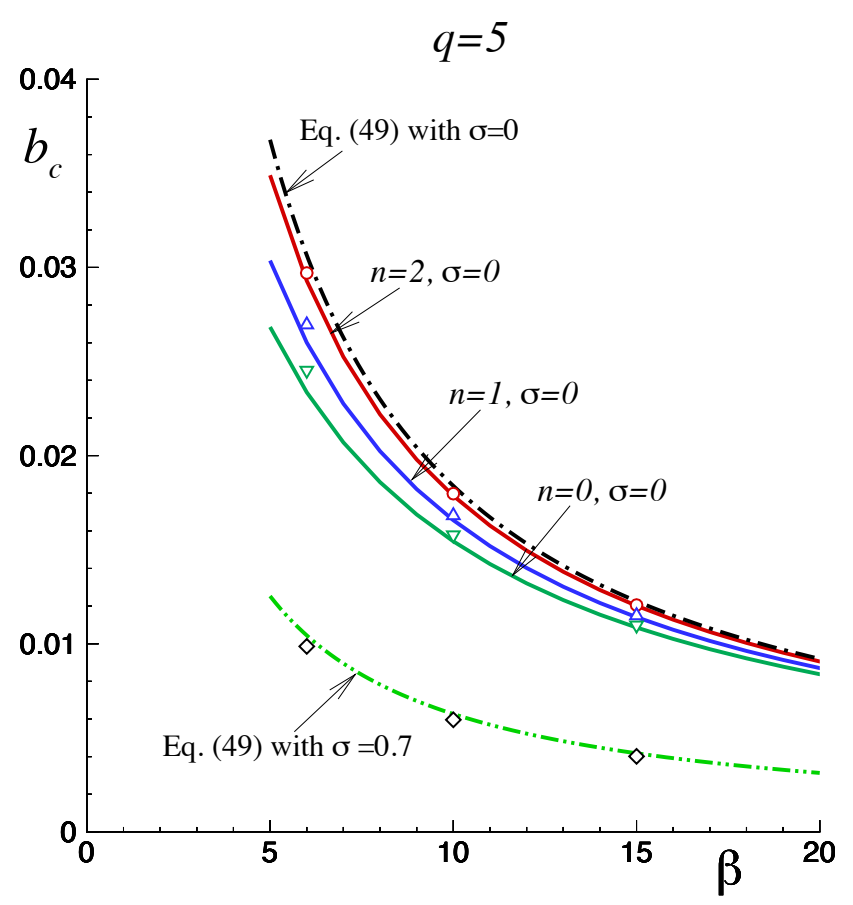

Figure 9: Critical values of heat-loss intensity, $b_{c}$, plotted versus $\beta$ for $q=5$ : solid lines asymptotic results based on Eqs. (42) and (21), for $\sigma=0$ and varios $n$; gradient, delta and open circle symbols - numerical results calculated from Eqs. (12)-(13) for $n=0,1$ and 2, respectively; diamond symbols - numerical results, for $\sigma=0.7$ and $n=1$; dash-dot and dash-dot-dot lines Eq. (49) for $\sigma=0$ and $\sigma=0.7$, respectively.

It is worth to emphasize that the expression for $A(\sigma, q)$ was obtained in an exact form given by Eq. (A7). One can see also that Eq. (48) switches to Eq. (44) for $\sigma=0$ because

$$
A(\sigma, q) \rightarrow 2-\sigma\left[1-q^{-1} \ln (1+q)\right]+O\left(\sigma^{2}\right) \quad \text { as } \quad \sigma \rightarrow 0 .
$$

Notice that in contrast to Eq. (44) the dependence on $q$ and $\sigma$ persists in Eq. (48). To the author's knowledge, the result given by Eqs. (47)-(48) for arbitrary $\sigma$ and $q$ was not reported heretofore.

The critical intensity of heat losses, $b=b_{c}$, above which there are no steady-state solutions is of great importance for applications. In the limit $\beta \rightarrow \infty$ resulted in Eq. (48) it is

$$
b_{c}=\frac{2}{A(\sigma, q)(1+q)^{\sigma}} \cdot \frac{1}{2 e \beta}
$$

For the asymptotic solution governed by Eqs. (42) and (21) the flame temperature at extinction is determined by the equation

$$
\frac{d}{d \theta_{f}}\left[\left(\theta_{f}^{-2}-1\right) u_{L}^{2}\left(\theta_{f}\right)\right]=0
$$


which is reduced to find the roots of

$$
\sum_{n=0}^{5} B_{n} \theta_{f}^{n}=0 .
$$

Coefficients $B_{n}$ are presented in Appendix A. The corresponding (positive) root gives the critical temperature and, thus, the flame velocity at extinction and $b_{c}$.

The critical values of the heat-loss parameter $b_{c}$ are shown in Fig. 9 as a function of $\beta$ for $q=5$ and two values of $\sigma$. The numerical (exact) points are shown with open symbols and the asymptotic results given by Eqs. (42) and (21) are plotted with solid lines for $\sigma=0$. The limiting curve $\beta_{c}=0.1839 \beta^{-1}$ corresponding to Eq. (44) is plotted with a dash-dot line. It is noticeable that the asymptotic results derived in the present study approach significantly better the exact (numerical) points capturing also the dependence on the density power $n$ of the initial Arrhenius kinetic. The influence of $\sigma$ is illustrated with a dash-dot-dot curve for $\sigma=0.7$ corresponding to Eq. (49) and the exact (numerical) values are shown with diamond symbols.

\section{Conclusions and discussions}

The current investigation presents the accurate asymptotic analysis for the problem of premixed flames propagation in narrow channels with heat-loses. Within the narrow channel approximation applied in the study, the formulation is equivalent to the issue of a planar isobaric flame front propagating in a quiescent mixture subject to (linear with temperature) heat-losses. The analysis was carried out using the Zeldovich number, $\beta=E\left(T_{a}-T_{0}\right) / T_{a}^{2}$, as a large parameter by means of the matched asymptotic expansions method. The asymptotic results were compared with the direct numerical calculations. The novelty of the present asymptotic study consists in presentation of the results in a parametric form. Comparison with the numerical calculations reveals that in this way applicability of the asymptotic results is widened up to relatively low values of the expansion parameter. It is demonstrated also that in the limit $\beta \rightarrow \infty$ the asymptotic results of the present work coincides with those presented in the past.

This point can be established clearly using the case $\sigma=0$. Finally, the asymptotic procedure of the current study have resulted into the relations between $u_{L}$ and $b$ in the form

$$
b=\mathcal{F}\left(\theta_{f}, \beta\right), \quad u_{L}=\mathcal{G}\left(\theta_{f}, \beta\right),
$$

where the function $\mathcal{F}$ and $\mathcal{G}$ are given by Eq. (21) and (42), respectively. Expanding the flame temperature for $\beta \gg 1$ as follows

$$
\theta_{f}=1-\frac{\theta_{f 1}}{\beta}-\frac{\theta_{f 2}}{\beta^{2}}-\frac{\theta_{f 3}}{\beta^{3}}+\ldots
$$


gives

$$
\begin{aligned}
& b=\frac{f_{1}\left(\theta_{f 1}\right)}{\beta}+\frac{f_{2}\left(\theta_{f 1}, \theta_{f 2}\right)}{\beta^{2}}+\frac{f_{3}\left(\theta_{f 1}, \theta_{f 2}, \theta_{f 3}\right)}{\beta^{3}}+\ldots, \\
& u_{L}=g_{0}\left(\theta_{f 1}\right)+\frac{g_{1}\left(\theta_{f 1}, \theta_{f 2}\right)}{\beta}+\frac{g_{3}\left(\theta_{f 1}, \theta_{f 2}, \theta_{f 3}\right)}{\beta^{2}}+\ldots .
\end{aligned}
$$

While the standard asymptotic treatment manages only the first terms in Eq. (54),

$$
b=\frac{f_{1}\left(\theta_{f 1}\right)}{\beta}, \quad u_{L}=g_{0}\left(\theta_{f 1}\right),
$$

leading to Eq.(44), the parametric representation given by Eq. (52) takes into account all terms entirely in the above expansions in such a manner extending applicability of the asymptotic results.

Re-writing the asymptotic formulae in the corresponding dimensional form gives the dependence of the reactant flux toward the reaction zone as a function of the flame temperature as follows

$$
-\left.\rho \mathcal{D} \frac{\partial Y}{\partial x^{\prime}}\right|_{x^{\prime}=0-}=\rho_{0} S_{L} \mu^{-1 / 2}(\alpha)\left(\frac{T_{f}}{T_{a}}\right)^{2+\frac{\sigma-n}{2}} \exp \left\{\frac{E}{2 \mathcal{R}_{g}}\left(\frac{1}{T_{a}}-\frac{1}{T_{f}}\right)\right\}
$$

where $S_{L}$ is the velocity of the planar adiabatic flame.

One can see that the right-hand side of Eq. (55) differs from that of Eq. (3) used the studies with the flame-sheet model adopted by a factor depending in the flame temperature. It is remarkable that Eq. (55) recovers the density dependence of the initial Arrhenius kinetic, namely parameter $n$ appearing Eq. (1). There is good reason to believe that the use of Eq. (55) will help to improve accuracy of flame-sheet model applications.

The classical result given by Eq. (44) obtained in the limit $\beta \rightarrow \infty$ was derived under conditions of constant transport properties. As a consequence, parameters $\sigma$ and $q$ determining variations of the heat conductivity with the temperature drop out of Eq. (44). This limitation has been relaxed in Eq. (48) obtained in the present study. It is interesting that coefficient $A(\sigma, q)$ was calculated in its exact form.

\section{Acknowledgments}

The present work is supported under the project \#ENE2015-65852-C2-2-R ( MINECO / FEDER, $\mathrm{EU})$. 


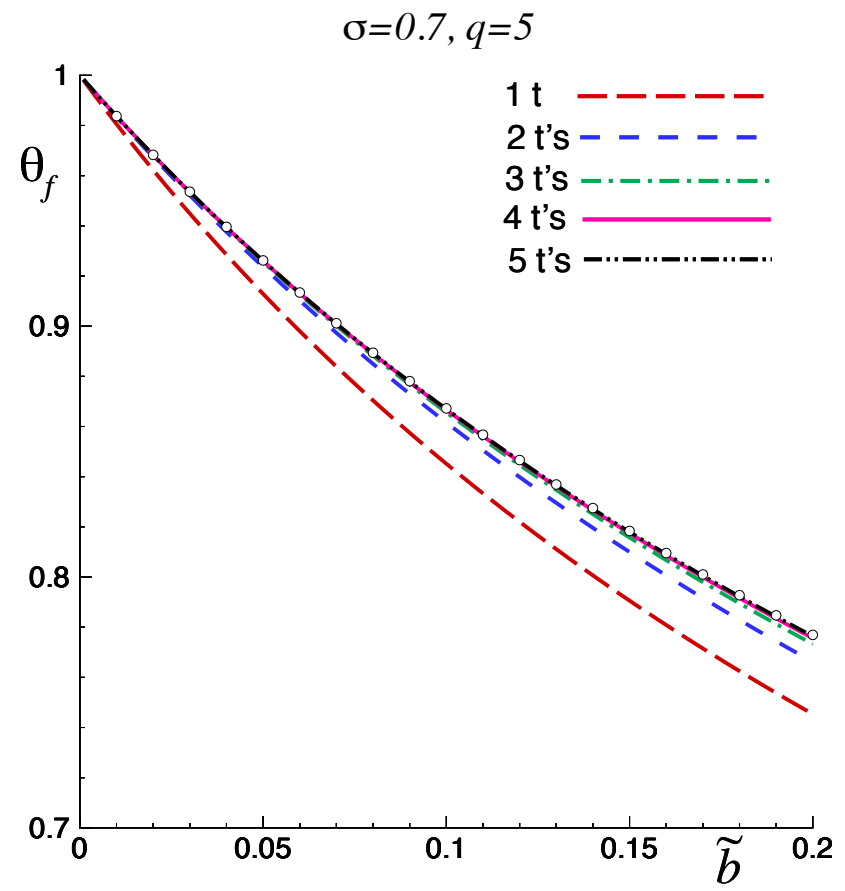

Figure 10: Variations of $\theta_{f}$ with $\tilde{b}$ plotted using from one- to five- terms in Eq. (A4); open circles - the numerical solution of Eqs. (A2)-(A3); all curve calculated with $\sigma=0.7$ and $q=5$.

\section{Appendix A. Treatment of the outer solution for arbitrary $\sigma$}

The following substitutions

$$
z=\frac{\theta}{\theta_{f}}, \quad F(z)=\frac{d z}{d \xi}
$$

transform Eq. (25) to

$$
F(z)=F(z) \frac{d}{d z}\left[\left(\frac{1+q \theta_{f} z}{1+q \theta_{f}}\right)^{\sigma} F(z)\right]-\tilde{b} z
$$

to be solved for $0<z<1$. The boundary conditions (26) take the form

$$
F(0)=0, \quad \theta_{f}\left[F^{(+)}(1)-F^{(-)}(1)\right]+1=0,
$$

where $F^{(-)}(z)$ and $F^{(+)}(z)$ denote the functions corresponding to the spans $-\infty<\xi<0$ and $0<\xi<\infty$, respectively. The functions $F^{( \pm)}(z)$ satisfying the same equation given by Eq. (A2) display different asymptotic behaviors

$$
z \rightarrow 0: \quad F^{( \pm)}=C^{( \pm)} z
$$




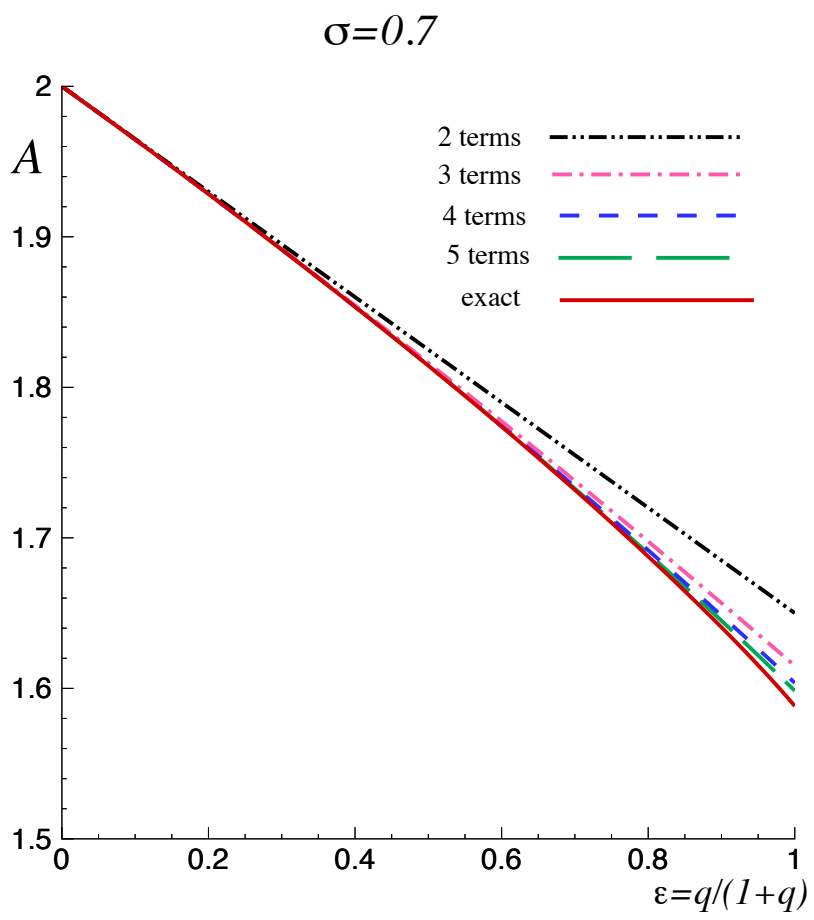

Figure 11: Coefficient $A$ appearing in Eq. (48) as a function of $\epsilon=q /(1+q)$; a solid line - the exact expression given by Eq. (A7).; all curves plotted for $\sigma=0.7$.

where

$$
C^{( \pm)}=\frac{\left(1+q \theta_{f}\right)^{\sigma} \mp \sqrt{\left(1+q \theta_{f}\right)^{2 \sigma}+4 \tilde{b}\left(1+q \theta_{f}\right)^{\sigma}}}{2}
$$

Equations (A2)-(A3) were solved numerically using $\theta_{f}$ as a shooting parameter. Derivatives of the dimensionless temperature at the flame sheet appearing in Eq. (33) are $\Phi^{( \pm)}=d \theta /\left.d \xi\right|_{\xi \rightarrow \pm 0}=$ $\theta_{f} F^{( \pm)}(1)$.

The approximate solution of Eqs. (A2)-(A3) was sought as a power series of $\epsilon=q /(1+q)$ in the form

$$
\begin{aligned}
& \theta_{f}=\theta_{f 0}+\epsilon \theta_{f 1}+\epsilon^{2} \theta_{f 2}+\epsilon^{3} \theta_{f 3}+\epsilon^{4} \theta_{f 4} \ldots, \\
& F(z)=F_{0}(z)+\epsilon F_{1}(z)+\epsilon^{2} F_{2}(z)+\epsilon^{3} F_{3}(z)+\epsilon^{4} F_{4}(z) \ldots
\end{aligned}
$$

Five terms were calculated in an analytic form by menas of the MAPLE facilities which simplifies the algebra tremendously. Although this leads to analytical expressions for all terms, writing them down does not reveal much about the nature of the solution. We present here only three 
terms of the flame temperature expansion:

$$
\begin{gathered}
\theta_{f 0}=\frac{1}{\sqrt{1+4 \tilde{b}}}, \quad \theta_{f 1}=\frac{(6 \tilde{b}+1) \tilde{b} \sigma}{144 \tilde{b}^{3}+104 \tilde{b}^{2}+25 \tilde{b}+2} \\
\theta_{f 3}=\frac{\left(c_{0}+c_{1} \cdot(1+4 \tilde{b})^{3 / 2}+c_{2} \sigma\right) \sigma \tilde{b}}{c_{3}(1+4 \tilde{b})^{3 / 2}}
\end{gathered}
$$

where

$$
\begin{gathered}
c_{0}=-2592 \tilde{b}^{4}-2124 \tilde{b}^{3}-641 \tilde{b}^{2}-84 \tilde{b}-4, \\
c_{1}=864 \tilde{b}^{3}+498 \tilde{b}^{2}+95 \tilde{b}+6, \\
c_{2}=576 \tilde{b}^{4}+26 \tilde{b}^{3}-91 \tilde{b}^{2}-24 \tilde{b}-2, \\
c_{3}=20736 \tilde{b}^{5}+23472 \tilde{b}^{4}+10600 \tilde{b}^{3}+2387 \tilde{b}^{2}+268 \tilde{b}+12 .
\end{gathered}
$$

All five terms can be obtained entirely using the MAPLE facilities. Figure 10 shows the variations of $\theta_{f}$ plotted using one, two, three, four and five terms in Eq. (A4) calculated with $\sigma=0.7$ and $q=5$. Open circles show exact numerical solution. One can see in Fig. 10 that the difference between three-, four- and five-terms expansions is practically negligible within range of $\tilde{b}$ considered.

Using the five-terms expansion (A4) calculated analytically gives

$$
\tilde{b} \rightarrow 0: \quad \theta_{f}=1-A \cdot \tilde{b}+O\left(\tilde{b}^{2}\right)+\ldots, \quad \Phi^{(+)}=-\tilde{b}+O\left(\tilde{b}^{2}\right)+\ldots,
$$

where

$$
A=2-\sigma\left[\frac{\epsilon}{2}+\frac{\epsilon^{2}(1-\sigma)}{6}+\frac{\epsilon^{3}(1-\sigma)(2-\sigma)}{24}+\frac{\epsilon^{4}(1-\sigma)(2-\sigma)(3-\sigma)}{120}+\ldots\right]
$$

was obtained also with the help of Maple facilities.

Only the terms written explicitly in Eq. (A6) were calculated analytically because the algebraic difficulties grow insuperably for the higher terms. Nontheless it is evident that the expansion appearing in Eq. (A6) can be continued as follows

$$
A=2-\sigma \sum_{n=1}^{\infty} \frac{\epsilon^{n}}{(n+1) !} C_{n, \sigma}, \quad \text { where } \quad C_{n, \sigma}= \begin{cases}\prod_{k=1}^{n-1}(k-\sigma) \equiv \frac{\Gamma(n-\sigma)}{\Gamma(1-\sigma)}, & n>1 \\ 1, & n=1\end{cases}
$$

and $\Gamma(z)=\int_{0}^{\infty} e^{-t} t^{z-1} d t$ is the Gamma function. After this guess, the above expression can be reduced (again using the Maple tools) to the following form

$$
A=2-\sigma\left[\frac{(1-\epsilon)^{\sigma+1}-1+\epsilon(\sigma+1)}{\sigma(\sigma+1) \epsilon}\right]
$$




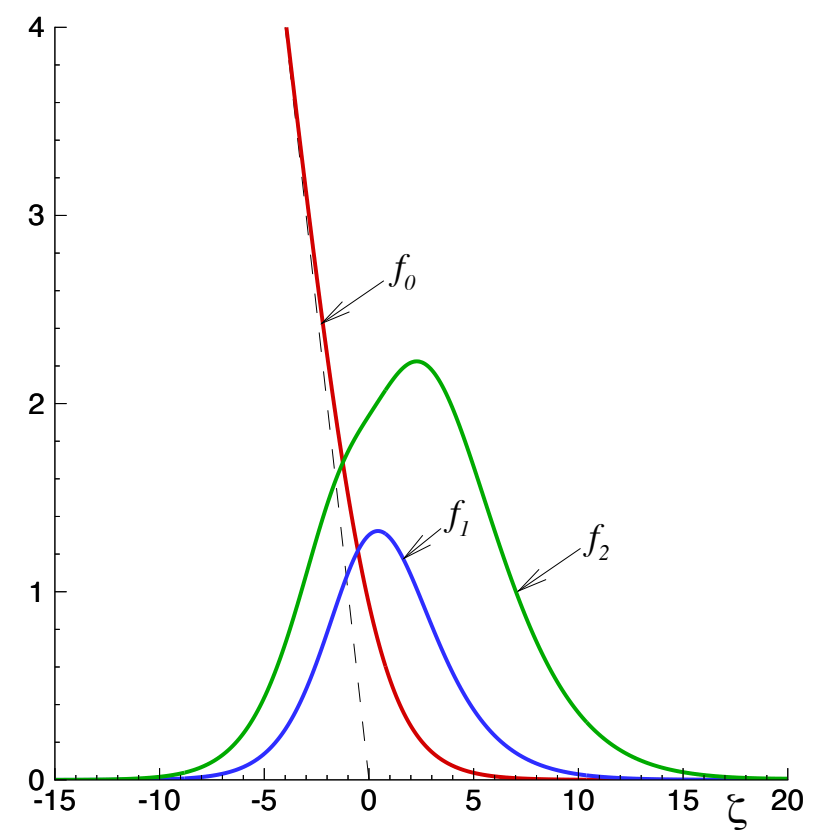

Figure 12: Functions $f_{0}(\zeta), f_{1}(\zeta)$ and $f_{2}(\zeta)$ appearing in Eq. (B1) calculated with $\mu_{1}=1.3440046$ and $\mu_{2}=-0.90323 ;$ a dashed line shows the asymptote $-\zeta$.

It is reasonably safe to suggest that Eq. (A7) constitutes an exact solution for coefficient $A$. Figure 11 compares various finite-terms expansions of $A$ and the exact expression given by Eq. (A7) for $\sigma=0.7$. Notice, that the interval $0<\epsilon<1$ corresponds to $0<q<\infty$.

The coefficients appearing in Eq. (51) with approximation $\mu(\alpha)=1-\mu_{1} \alpha$ used are

$$
\begin{gathered}
B_{5}=\mu_{1} q^{2}(3-n) \\
B_{4}=q^{2}(8-2 n)+\mu_{1}\left[(\beta+n-4) q^{2}+(2 \beta-n+2) q+\beta\right] \\
B_{3}=\left[2 \beta q^{2}+(4 \beta-2 n+8) q+2 \beta\right]+\mu_{1}\left[(n-\beta-1) q^{2}+(n-2 \beta-4) q-(1+\beta)\right] \\
B_{2}=(2 n-4) q^{2}+\mu_{1}\left[(-\beta-n+2) q^{2}+(-2 \beta+n+2) q-\beta\right] \\
B_{1}=\left[-2 \beta q^{2}+(2 n-4 \beta) q-2 \beta\right]+\mu_{1}\left[\beta q^{2}+(2 \beta-n) q+(\beta+3)\right] \\
B_{0}=4-\mu_{1}
\end{gathered}
$$




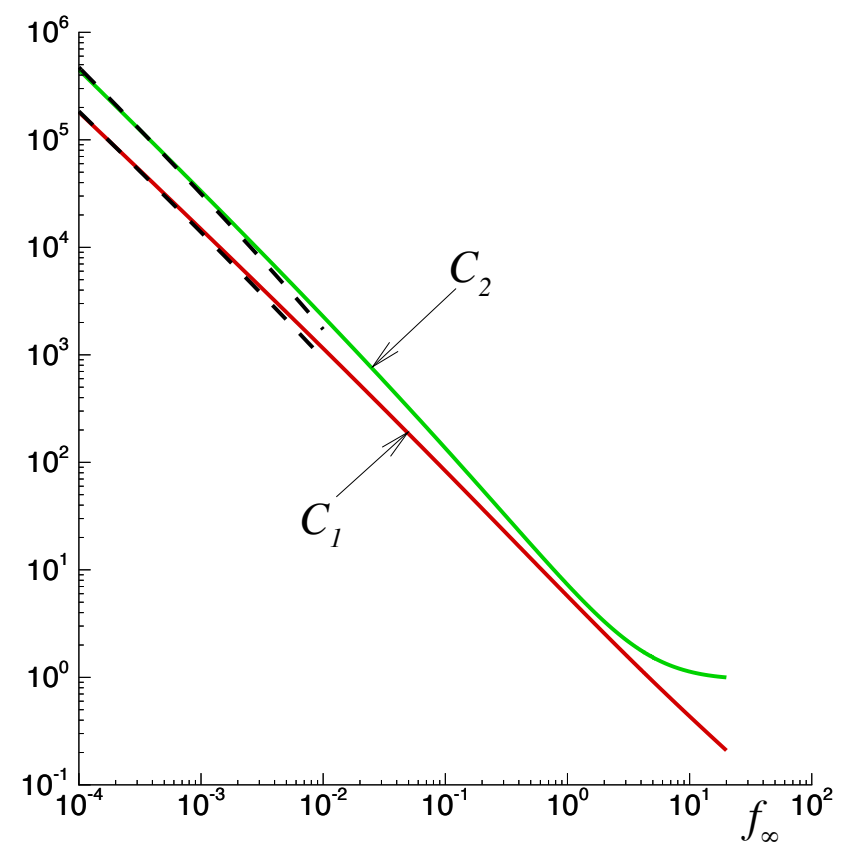

Figure 13: $C_{1}$ and $C_{2}$ appearing in Eq. (40) as function of $f_{\infty}$.

\section{Appendix B. Inner solution for $|\alpha| \ll 1$}

In is well known that for $\alpha=0$ equations (36)-(37) give $\mu=1$. For $|\alpha| \ll 1$ the solution was obtained in power series of $\alpha$ in the form

$$
f=f_{0}(\zeta)+\alpha f_{1}(\zeta)+\alpha^{2} f_{2}(\zeta) \ldots, \quad \mu=1-\mu_{1} \alpha-\mu_{2} \alpha^{2}+\ldots
$$

Functions $f_{0}$ and $f_{1}$ satisfy the equations

$$
\begin{gathered}
\frac{d^{2} f_{0}}{d \zeta^{2}}=\frac{1}{2} f_{0} e^{-f_{0}}, \quad \frac{d^{2} f_{1}}{d \zeta^{2}}=\frac{1}{2}\left(f_{1}-\mu_{1} f_{0}\right) e^{-f_{0}}-\frac{1}{2} f_{0}\left(f_{1}+\zeta\right) e^{-f_{0}} \\
\frac{d^{2} f_{2}}{d \zeta^{2}}=\frac{1}{2} f_{0} e^{-f_{0}}\left(-f_{2}+\frac{1}{2} f_{1}^{2}+f_{1} \zeta+\frac{\zeta^{2}}{2}\right)+\frac{1}{2}\left(f_{2}-\mu_{2} f_{0}-\mu_{1} f_{1}\right) e^{-f 0} \\
-\frac{1}{2}\left(f_{1}-\mu_{1} f_{0}\right)\left(f_{1}+\zeta\right) e^{-f 0}
\end{gathered}
$$

to be solved subject to

$$
\zeta \rightarrow-\infty: \quad f_{0}+\zeta, f_{1}, f_{2} \rightarrow 0 ; \quad \zeta \rightarrow+\infty: \quad f_{0}, f_{1}, f_{2} \rightarrow 0
$$

The numerical solution shown in Figure 12 was calculated using a shooting method and $\mu_{1} \approx$ $1.3440046, \mu_{2} \approx-0.90323$ were obtained. These values coincide very well with those which can be obtained from the correlation suggested in [18]. 
The numerical values of $C_{1}$ and $C_{2}$ appearing in Eq. (40) are plotted in Fig. 13 with solid lines. The corresponding asymptotic approximations can be written in the form

$$
C_{1} \rightarrow 2 f_{\infty}^{-1} \ln \left(1 / f_{\infty}\right), \quad C_{2} \rightarrow\left\{\ln \left[\ln \left(1 / f_{\infty}\right)\right]+\ln \sqrt{2}\right\} \cdot C_{1} \quad \text { as } \quad f_{\infty} \rightarrow 0+.
$$

These dependencies are indicated in Fig. 13 with dashed lines. Consequently $f_{\infty}=e^{-1 / \alpha}$ is obtained as $\alpha \rightarrow 0+$ plotted in Fig. 3 (right) with a dashed line.

\section{References}

[1] W.B. Bush, F.E. Fendell, Asymptotic analysis of laminar flame propagation for general Lewis number, Combust. Sci. Tech. 1 (1970) 421-428.

[2] Y.B. Zeldovich, D.A. Frank-Kemenetskii, Thermal theory of flame propagation, Zhur. Fiz. Khim. 12 (1938) 100-105.

[3] Ch. K. Law, Combustion physics, Cambridge Universuty Press, 2006.

[4] D.B. Spalding, A theory of inflammability limits and flame-quenching, Proc. Roy. Soc. London Ser. A 240 (1957) 83-100.

[5] G. Joulin, P. Clavin, Analyse asymptotique des conditions d'extinction des flammes laminaires, Acta Astr. 3 (1976) 223-240.

[6] J.D. Buckmaster, The quenching of deflagration waves, Combust. Flame 26 (1976) 151162.

[7] J.D. Buckmaster, G.S.S. Ludford, Theory of laminar flames, Cambrige University Press, new York, 1982.

[8] G.I. Sivashinsky, The diffusion stratification effect in Bunsen flames, J. Heat Hransfer. ASME Transactions 96 (1974) 530-535.

[9] G.I. Sivashinsky, Diffusion-thermal theory of cellular flames, Combust. Sci. Tech. 15 (1977) 137-146.

[10] J. Buckmaster, G. Joulin, P. Ronney, The strusture and stabity of nonadiabatic flame balls, Combust. Flame 79 (1990) 381-392.

[11] S. Minaev, L. Kagan, G. Joulin, G. Soivashinsky, Combust. Theory Modell. 5 (2001) 609622. 
[12] S. Minaev, R. Fursenko, C.K. Law, Stability analysis of near-limit stretched premixed flames, J. Fluid Mech. 488 (2003) 225-244.

[13] J.C. Graña-Otero, Nonlinar dynamics of unsteady premixed, planar flames, Universidad Politécnica de Madrid, 2009, Doctoral thesis.

[14] V.N. Kurdyumov, M. Matalon, Flame acceleration in long narrow open channels, Proc. Comust. Inst. 34 (2013) 865-872.

[15] M.D. Smooke, V. Giovangigli, Formulation of the premixed and non-premixed test problems, in: M.D. Smooke (Ed.), Reduced Kinetic Mechanisms and Asymptotic Approximations for MethaneAir Flames, Lecture notes in physics, vol. 384, Springer-Verlag (1991), pp. 1-28.

[16] V.N. Kurdyumov, M. Matalon, Self-accelerating flames in long narrow open channels, Proc. Comust. Inst. 35 (2015) 921-928.

[17] V.N. Kurdyumov, M. Matalon, Effects of gas compressibility on the dynamics of premixed flames in long narrow adiabatic channels, Combust. Theory Modell. 20 (2016) 1046-1067.

[18] A. Liñán, The asymptotic structure of counterflow diffusion flames for large activation energies, Acta Asronautica, 1 (1974) 1007-1039. 